\title{
Editorial: Topical Collection on Venus
}

\author{
Bruno Bézard ${ }^{1}$. Christopher T. Russell ${ }^{2}$. \\ Takehiko Satoh $^{3}$. Suzanne E. Smrekar ${ }^{4}$. \\ Colin F. Wilson ${ }^{5}$
}

Published online: 27 November 2018

(C) Springer Nature B.V. 2018

The incredible diversity of scientific questions that can be investigated at Venus, Earth's twin planet, is manifested by the rich history of exploration and breadth of surface, interior, and atmospheric studies. This Venus III collection of papers discusses the current understanding of Venus following the end of the Venus Express mission in December 2014. The pioneering book Venus (Hunten, Colin, Donahue, Moroz Eds.), published in 1983, reported on the amazing set of data collected by a series of Mariner, Venera and Pioneer Venus missions between 1962 and 1978, including several surface landers. The Venus II book (Bougher,

Venus III

Edited by Bruno Bézard, Christopher T. Russell, Takehiko Satoh, Suzanne E. Smrekar and Colin F. Wilson

\section{B. Bézard \\ bruno.bezard@obspm.fr \\ C.T. Russell \\ ctrussel@igpp.ucla.edu}

T. Satoh

satoh@stp.isas.jaxa.jp

S.E. Smrekar

suzanne.e.smrekar@jpl.nasa.gov

C.F. Wilson

colin.wilson@physics.ox.ac.uk

1 LESIA, Observatoire de Paris, Université PSL, CNRS, Sorbonne Université, Univ. Paris-Diderot, Sorbonne Paris Cité, 92195 Meudon, France

2 Earth Planetary and Space Sciences, University of California, Los Angeles, CA 90095, USA

3 Department of Solar System Sciences, Institute of Space and Astronautical Science, JAXA, 3-1-1 Yoshinodai, Chuo-ku, Sagamihara, Kanagawa, 252-5210, Japan

4 Geophysics \& Planetary Geosciences, Jet Propulsion Laboratory, California Institute of Technology, 4800 Oak Grove Drive, Pasadena, CA 91109, USA

5 Department of Physics, University of Oxford, Parks Road, Oxford OX1 3PU, UK 
Hunten and Phillips Eds.) that came out in 1997 was motivated by the need to reassess the state of our knowledge following the end of the Pioneer Venus Orbiter in 1992, more USSR missions (Venera and Vega), the Galileo flyby in 1990, the Magellan mission (19901994), and various ground-based observational campaigns. Since then, the Venus Express spacecraft, which was launched in November 2005 and ceased activity in December 2014, has brought a wealth of unique data on Venus' atmosphere, surface and space environment. With this major landmark in the history of Venus's exploration, the time was ripe for synthesizing the resulting scientific studies together with other advances in a Venus III book, this Topical Collection of Space Science Reviews. This collection comes too early to incorporate all the findings from the Akatsuki orbiter, which arrived in December 2015 and is conducting its observations of Venus as this set of papers goes to press.

The ten articles in this collection are the direct products of the efforts of 41 authors and co-authors. They review our knowledge of Venus's interior structure, surface composition, and atmosphere in terms of thermal structure, dynamics, composition, chemistry, clouds, aeronomy and interaction with the solar wind. They also identify remaining questions and needed measurements, which provide fuel for thinking about the future of Venus research and exploration.

As an introduction to the following articles, the paper by Taylor et al. presents a brief history of Venus exploration and an overview for the general reader of current knowledge on the surface, interior, atmosphere, climate and magnetosphere of Earth's near twin planet. The paper by Smrekar et al. reviews current data that constrain interior structure and dynamics, and provides new insights into key questions, such as why plate tectonics is present on Earth and absent on Venus. Gilmore et al. review the constraints on Venus's surface composition derived from Venus Express near-infrared measurements along with the recent theoretical and experimental works that improve our understanding of atmosphere-surface interactions. The paper by Limaye et al. is devoted to the thermal structure and radiative balance of Venus's atmosphere. Recent results on the vertical thermal structure from different remote sensing techniques are presented with emphasis on knowledge gaps and open issues. The atmospheric dynamics of Venus from the surface up to $\sim 100 \mathrm{~km}$ is reviewed by Sanchez-Lavega et al. The paper includes descriptions of the wind field, dominated by the so-called super-rotation, of the prominent polar vortices, and of the various types of waves that have been detected. Numerical models that have been developed to investigate Venus's global circulation are also reviewed. Marcq et al. discuss the composition and chemistry of the neutral atmosphere, focusing on the horizontal, vertical and temporal variability of the minor species observed below $\sim 90 \mathrm{~km}$. Particular attention is paid to the vertical profiles of $\mathrm{CO}$ and OCS below the clouds and of $\mathrm{CO}, \mathrm{SO}$ and $\mathrm{SO}_{2}$ above the clouds. The paper by Titov et al. explores the complexity of the Venus cloud system. After a presentation of the vertical structure of the mesospheric hazes and main cloud deck, the paper reviews the available information on the cloud morphology and on the microphysical properties of the particle population, discusses the cloud composition, chemistry and microphysics, describes the radiative effects of the cloud layers and evaluates the occurrence of lightning. The paper by Gérard et al. reviews the composition, thermal structure, dynamics and transport of the Venus upper atmosphere above $\sim 90 \mathrm{~km}$. It describes in particular how observations of the dayglow and nightglow emissions from different molecules provide invaluable tools to sound this region and improve three-dimensional models of its dynamics. The paper by Futaana et al. examines the near-Venus space environment, the solar wind interaction with Venus and its impacts down to the ionopause. Emphasis is put on results from the Venus Express mission, which observed with unprecedented detail the structure of the plasma environment, particularly the north polar region, revealed the neutral exosphere, and provided a measurement of the water loss from planetary ion escape. 
The collection concludes with the paper by Glaze et al., which opens a perspective towards the future of Venus research and exploration. Many key questions remain about Venus's evolution and current geologic and atmospheric processes. Their elucidation will require laboratory and modeling studies as well as new measurements from Earth and spacecraft, including new missions capable of making in situ measurements in the atmosphere and at the surface.

The success of this collection has been due to many people. First of all, the editors wish to thank the authors who had the difficult job of distilling the voluminous reports that such missions produce, to a coherent set of highly readable articles. The editorial process also benefited from an excellent group of referees who acted as a test readership and helped guide the authors to produce even more complete and useful manuscripts. These referees included Sushil K. Atreya, Bruno Bézard, Mark Bullock, Thérèse Encrenaz, Justin Filiberto, Misha Ivanov, Esa Kallio, Vladimir Krasnopolsky, Emmanuel Lellouch, Helen Parish, Javier Peralta, Peter Read, Takehiko Satoh, Gerald Schubert, Tom G. Slanger, Suzanne Smrekar, Paul Tackley, Adriana C. Ocampo Uria, O.L. Vaisberg.

Equally important has been the strong support this project has received from Springer Nature and the extra effort expended by Ganesh Jagadeesan, Vygintas Vilimas, and Hannah Kaufman. At UCLA we were skillfully assisted by Marjorie Sowmendran who acted as the interface between the authors, the referees, and the publishers. 